East African Medical Journal Vol. 83 No. 11 November 2006

ROUTINE USE OF ANTIMICROBIAL DRUGS DURING THE 2004 CHOLERA EPIDEMIC IN DOUALA, CAMEROON J. Noeske, MD, PhD, German Technical Cooperation (GTZ), Technical advisor to the Provincial Delegation of Public Health in the Littoral Province, BP 4400, Douala, Cameroon, E. Guévart, MD, Institut Regionale de Sante Publique de Ouidah, BP. 918, Cotonou, Benin, C. Kuaban, MD, MPH, Professor, University of Yaounde 1, BP. 1274, Yaoundé, Cameroon, J. Solle, MD, Provincial Delegation of Public Health of the Littoral Province, P.B. 4012, Douala, Cameroon, M-C Fonkoua, Biologist, Centre Pasteur de Cameroon, B.P. 1274, Yaounde, Cameroon, A. Mouangue, MD, Provincial Delegation of Public Health, P.B. 4012, Douala, Cameroon and A.B. Fouda, MD, Provincial Delegation of Public Health, P.B. 4012, Douala, Cameroon

Request for reprints to: Dr. J. Noeske, German Technical Cooperation (GTZ), Technical advisor to the Provincial Delegation of Public Health in the Littoral Province, BP 4400, Douala, Cameroon

\title{
ROUTINE USE OF ANTIMICROBIAL DRUGS DURING THE 2004 CHOLERA EPIDEMIC IN DOUALA, CAMEROON
}

\author{
J. NOESKE, E. GUÉVART, C. KUABAN, J. SOLLE, M-C. FONKOUA, A. MOUANGUE and A.B. FOUDA
}

\begin{abstract}
Objectives: To evaluate routine use of antimicrobial drugs for treatment and prevention of cholera with special regards to the evolution of the antimicrobial drug resistance patterns of V.cholerae strains. Design: Retrospective population-based descriptive study.

Subjects: Four thousand nine hundred and forty one notified cholera cases, their 15,381 patients' guards and their 159,263 household members and close neighbours.

Results: A total of 4,941 patients received antibiotic therapy according to the treatment protocols. Prophylactic treatment was administered to 15,381 patients' guards in hospitals and to 159,263 household members and close neighbours during home visits. Over the entire outbreak, the antimicrobial susceptibility patterns of $V$. cholerae strains isolated remained stable.

Conclusions: The routine use of antimicrobial therapy for cholera cases associated with simultaneous and large scale chemoprophylaxis of close contacts does not seem in our experience to compromise the stability of $V$. cholerae susceptibility profiles to drugs when applied within a comprehensive package of rigorously monitored community interventions. The role of therapy and chemoprophylaxis in limiting the extent of a cholera epidemic is however difficult to ascertain from our experience. Field trials need to be designed to elucidate this aspect.
\end{abstract}

\section{INTRODUCTION}

A general rule stipulates that prolonged use of the same antibiotic should be avoided and that for prevention another antibiotic than the one administered for curative care should be used, all in a bid to avoid the emergence of resistant bacterial strains (1). Vibrio cholerae, a Gram-negative bacillus, is usually susceptible to various antibiotics. In cholera treatment, antimicrobial therapy can shorten the faecal excretion of $V$. cholerae and lessen diarrhoeal purging (2). There is also evidence that healthy cholera carriers may play a role in the transmission of cholera infection and that cholera contacts preventively treated may result in a reduction in the disease incidence (3). Prophylactic antibiotics given to cholera contacts may therefore limit the spread of an epidemic. In various settings, however, mass prevention with antimicrobial therapy dispensed during cholera epidemics seemed to have provoked the emergence of resistant strains of $V$. cholerae $(4,5)$. As such, strategies for the control of cholera outbreaks as recommended by the World Health Organisation (WHO) do not include the use of antibiotics for mass prophylaxis (6). 
In early January 2004, cases of severe watery diarrhoea were reported in Douala, the economic capital of Cameroon with an estimated population of 2.4 million inhabitants. The first three stool samples of patients examined at Centre Pasteur du Cameroun (CPC), the country's National Reference Laboratory, grew $V$. cholerae serogroup 01, later identified by Institut Pasteur in Paris, France, as serotype Inaba. Antimicrobial susceptibility testing of these strains revealed that they were resistant to trimethoprim-sulfamethoxazole and colistine but susceptible to amoxicillin and doxycycline. An outbreak of cholera which lasted about eight months was declared by the country's health authorities.

In order to control the epidemic, a task force was set up at provincial level to plan and coordinate the various interventions to be applied. Strategies adopted by the task force included opening of cholera treatment units (CTU) in Douala hospitals, use of standardised treatment protocols including routine use of antimicrobial drugs and preventive treatment of contacts. In this paper we report our experience with routine use of antimicrobial drugs for treatment and prevention of cholera with special regards to the evolution of the antimicrobial resistance patterns of $V$. cholerae strains isolated during this 2004 cholera epidemic in Douala.

\section{MATERIALS AND METHODS}

Fourteen CTUs in hospitals equally distributed over the town of Douala were instructed by the task force to directly admit and treat free of charge any person who presented with symptoms suggestive of cholera. The diagnosis of cholera was made on clinical grounds according to WHO (6) recommendations by each physician in charge of a CTU. Treatment management protocols including rehydration and systematic prescription of antimicrobial therapy have already been described elsewhere by Guévart et al $(7,8)$. Taking into consideration (a) the antimicrobial susceptibility patterns of the $V$. cholerae strains isolated at the onset of the epidemic; (b) the fact that a single dose of doxycycline has been shown to reduce diarrhoeal discharge by patients as well as reduce cholera infection in contacts (9); (c) the possibility that treatment compliance could be ensured by a single dose or a short course regimen of antibiotherapy; and (d) the availability and tolerance as welI as low cost of drugs to be prescribed, it was decided that doxycycline or amoxicillin will be administered for therapy as well as for prevention. Antibiotics were administrated orally to patients as soon as vomiting ceased. A single dose of doxycycline at $5 \mathrm{mg} / \mathrm{kg}$ body weight was administered when there was no contraindication. Amoxicillin at $50 \mathrm{mg} / \mathrm{kg}$ body weight divided into three daily doses for three consecutive days was prescribed to pregnant or breastfeeding women and patients aged eight years or less. When a pregnant woman presented with persisting vomiting, oral amoxicillin was initially replaced by injectable ampicillin. Patients' characteristics and treatments received by them were recorded in cholera treatment unit registers and on individual patients' treatment cards.

The antibiotic treatment regimens were as well systematically given as prophylaxis to patients' guards (i.e. family members taking care of their patients in hospital). Furthermore during systematic home visits to disinfect patients' homes and provide health education, all household members and close neighbours defined as people living directly in adjacent houses to the patients were equally given the same prophylaxis. Characteristics of persons treated prophylactically were recorded on adapted forms. Tight daily monitoring of the CTUs by supervisors and daily field visits ensured that diagnostic and curative algorithms as well as rules for prescribing and administering prophylaxis were correctly applied.

To ascertain the correctness of the diagnosis and thus the persistence of the cholera epidemic as well as monitor changes in the patterns of drug resistance of the $V$. cholerae strains isolated with the eventuality to modify the antimicrobial protocols used, stool samples of patients were collected through a convenience sampling procedure from the 14 CTUs. Five samples at least were collected each week. Collection of samples from diagnostically doubtful cases was encouraged. The stool specimens were collected from patients in sterile wide mouthed containers immediately after admission in the CTUs and prior to the administration of antibiotics and kept at $4-8^{\circ} \mathrm{C}$ in a refrigerated isothermic box. They were later transferred within 48 hours to the CPC for culture and antimicrobial drug susceptibility testing.

In the CPC, $V$. cholerae was searched for using conventional techniques including direct 
examination of wet mounts. Gram staining of fixed smears followed by culture and successive enrichment on alkaline peptone water. The media used for the isolation of $V$. cholerae were thiosulfate citrate bile salts sucrose agar (TCBS) and gelatine agar. Hektoin and Muller Kaufman agar were also used as primary culture media for the isolation of salmonellae and shigellae. Identification of $V$. cholera was based on morphologic, cultural and biochemical characteristics and its confirmation was based on an agglutination test using polyvalent 01 serum. Voges Proskauer (VP) reaction and sensitivity to polymixine were used to determine the biotype.

The disk-diffusion method recommended by the "Comité de l'Antbiogramme de la Société Française de Microbiologie" (10) was used to determine the susceptibility of the isolated $V$. cholerae strains to different antibiotics. The following antibiotics were tested: amoxicillin, amoxicillin + clavulanic acid, cefotaxime, chloramphenicol, colistin, doxycycline, gentamicin, nalidixic acid, perfloxacin, trimethoprimsulfamethoxazole and the vibriostatic agent 0/129. Four per cent of the isolated strains were randomly selected preserved in a conservation medium (BioRad, Maine la Coquette, France) and sent to Institut Pasteur in Paris, France for quality control, serotyping and coding for the cholera toxin gene and ribotyping.

\section{RESULTS}

A total of 4,941 patients with cholera were registered over the period of the epidemic. Of the 4,941 patients
2,725 (55\%) were male and 2,196 (45\%) female with a mean age of 27.9 years (range 1-83 years). All the patients received antibiotic therapy according to the standardised treatment guidelines set out at the onset of the epidemic. Sixty four cases of death were registered.

Prophylactic antibiotic treatment was given to 15,381 patients' guards averaging about three hospital contacts per patient. Visits were made to the homes of 4,130 ( $84 \%$ ) of the 4,941 cholera patients on the day of or the day after their hospitalisation. During these home visits a total of 159,263 contacts (an average of 32 community contacts per case) were given prophylactic antibiotic treatment. On the whole, 179,585 curative and prophylactic treatments were administered over the period of the epidemic. Table 1 presents details of the numbers of patients and contacts treated as well as the type of antibiotic used.

Growth of $V$. cholerae 01 strains was obtained from cultures of stool specimens for 81 (57\%) of the 141 patients whose stool samples were collected over the period of the epidemic. The rate of positive stool specimens for $V$. cholerae varied from month to month between $35 \%$ and $93 \%$ (Figure 1) and between hospitals from $33 \%$ to $71 \%$ (data not shown). No salmonella spp. were isolated but on one sample a strain of Shigella flexneri was found.

During the entire epidemic, the susceptibility patterns to antimicrobial drugs of the $V$. cholerae strains isolated remained stable. As from the onset of the outbreak, $V$. cholerae strains isolated during the course of the epidemic showed resistance to

Figure 1

Number of culture results (positive/negative) for $V$. cholerae per month

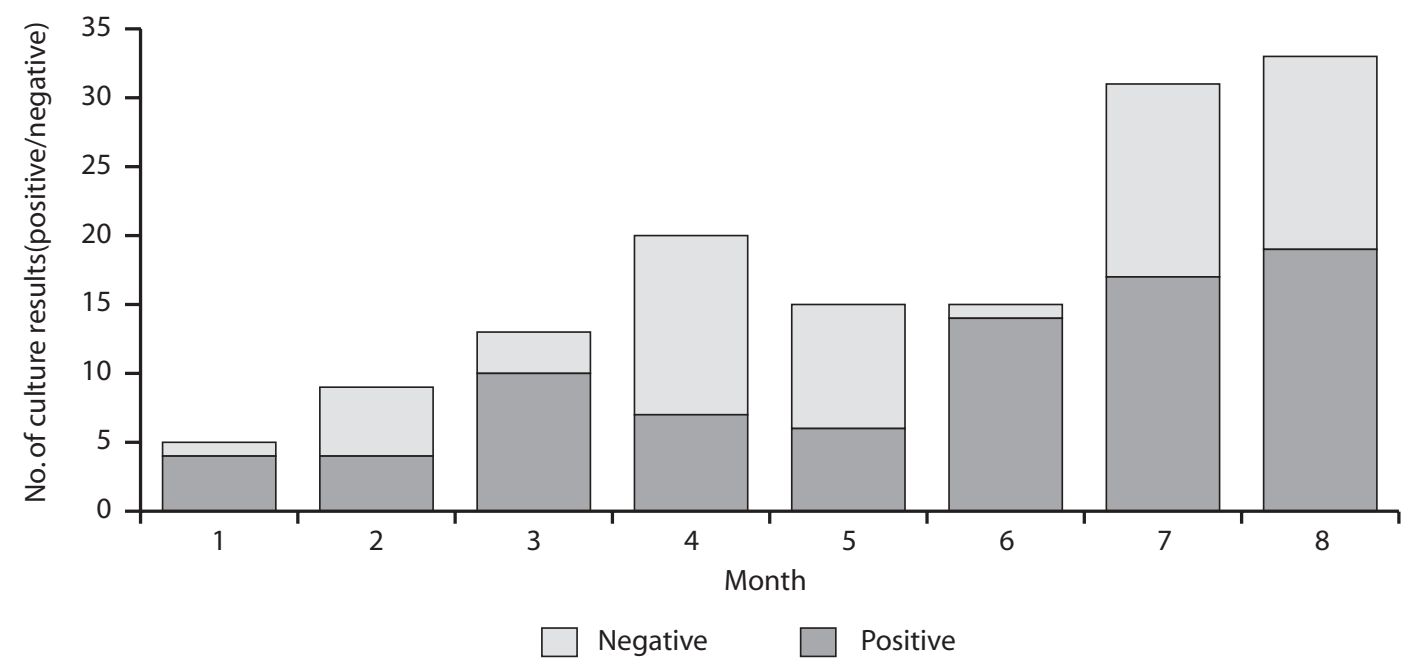


Table 1

Number of persons treated, type of antibiotic used, and patient-contact ratios

\begin{tabular}{lcccr}
\hline & \multicolumn{4}{c}{ Type of antibiotic } \\
Number of persons treated & Doxycycline & Amoxicillin (tablets) & Amoxicillin (syrup) & Total \\
\hline Patients in CTU & 4,506 & 416 & 19 & 4,941 \\
Contacts in CTU & 15,239 & 116 & 26 & 15,381 \\
Contacts in the community & 143,684 & 12,425 & 3,154 & 159,263 \\
\hline Total number treated & 163,429 & 12,957 & 3,199 & 179,585 \\
\hline Ratio contacts/patients & 3.1 & 0.25 & 1.4 & 3.1 \\
Ratio community contacts/patients & 31.9 & 29.8 & 168.6 & 32.2 \\
\hline
\end{tabular}

trimethoprim-sulfamethoxazole and colistine, intermediate resistance to chloramphenicol and susceptibility to amoxicillin, doxycycline, clavulanic acid, cefotaxime, doxycycline, gentamicin, nalidixic acid and perfloxacin.

\section{DISCUSSION}

Our case definition of cholera both clinically and epidemiologically was based on WHO's (6) recommendations for cholera epidemics. In order to ensure accuracy of the clinical diagnosis, the CTUs were closely monitored. During the epidemic, exhaustive sampling of cholera cases for bacteriological confirmation of the diagnosis was neither possible nor is it recommended (6). Our sampling strategy however permitted us to monitor for the presence of $V$. cholerae in patients' stools, ascertain the susceptibility patterns of isolates obtained to different antibiotics as well as detect other germs with an epidemic potential throughout the epidemic. Although the rate of positive samples may have been greater with better sampling of cases as well as better conservation and transport conditions of stool specimens, the presence of $V$. cholerae in almost $60 \%$ of the samples assured us of a persisting cholera epidemic. Despite these shortcomings, the positivity rate of our samples was relatively higher than the $42 \%$ and $50 \%$ rates reported in similar studies from Madagascar and Togo, respectively $(11,12)$.

Routine use of adjunctive antibiotic therapy is not recommended during cholera epidemics even though treatment with an effective antimicrobial drug for cases of suspected cholera with severe dehydration can be considered good clinical practice (2). This notwithstanding, we assumed that by reducing the volume and duration of cholera-related diarrhoea by antimicrobial therapy, we would diminish the need for rehydrating with intravenous fluids, shorten the average hospital stay, and render the excreta and vomitus of patients manipulated in our setting mostly by family members less infectious and consequently less contagious.

Cholera is transmitted by several distinct mechanisms. The predominant route of transmission depends largely on the degree of sanitation already achieved in a given setting. In Douala, attack rates of cholera were highest in densely populated precarious sub-urban areas with very poor access to safe drinking water. Indeed, the only public water provider in the town serves less than one fifth of her population. An average household in these densely populated areas of the town comprises between seven and ten persons. Very often several households share the same courtyard, a traditional kitchen and a pit latrine. The town, besides, does not dispose of an appropriate sewage drainage system. More than $90 \%$ of surface water originating from an intensive nine-months rainy season as well as waste water from households including that from latrines is evacuated through a network of open-air drains formed by the town's seven small rivers and their numerous tributaries in a swampy and low lying terrain that is practically at sea level. Before arriving in the main drains, the waste water from several neighbourhoods is channelled and drained through gutters along narrow foot paths leading to homes. More than $50 \%$ of the population of the town, composed mostly of young, unemployed and 
extremely mobile persons, work in the informal sector and generally eat meals sold along road-sides. Under these circumstances we assumed that we could rapidly and efficaciously shorten the period during which cases and asymptomatic vibrion carriers could contribute to transmission of the disease through the ejection of their excreta in an open and unprotected environment as described above by giving prophylaxis even though mass chemoprophylaxis is generally not recommended as a strategy to control an outbreak of cholera. Two main reasons are given against the use of chemoprophylaxis during a cholera outbreak. Firstly, prophylactic use of antibiotics is believed to greatly increase the risk of developing drug resistance by the germs. Secondly, it is doubtful whether the use of mass chemoprophylaxis is costeffective since the period covered by prophylactic treatment does not exceed 12 days (9). WHO (6), however, considers that selective chemoprophylaxis could be potentially useful for close contacts or in closed populations. We adopted this WHO concept of selective chemoprophylaxis in the Douala cholera epidemic but given the town's environmental and social context we considered all the inhabitants of a complex of adjacent households in which a case of cholera occurred as close contacts. We thought this approach was reasonable and justified as we gave chemoprophylaxis as part of a comprehensive package of community interventions that included health education, disinfection of latrines and wells in all the affected households. The success of this approach depended on pre-set conditions which included a clear definition of a close contact, prompt reaction of the intervention teams and rigorous application and monitoring of prevention procedures. The fact that the drug susceptibility patterns of $V$. cholerae did not change within the period of the epidemic as feared seems to justify retrospectively this practical approach.

The dynamics of cholera epidemics depend on a multitude of factors amongst which are the virulence of $V$. cholerae, the environment including access to clean drinking water and sanitary infrastructures, the degree of immunity in the population as well as the behaviour patterns of the population with regards to personal and nutritional hygiene and finally prophylactic and therapeutic interventions. The 2004 cholera epidemic in Douala lasted 31 weeks and had two epidemic peaks: one during the dry season at week four and the second, markedly more intense, during the first period of the rainy season after 20 weeks. A total of 4,941 cases were notified, with up to a hundred times more individuals infected (2). The population at risk when considering the number of inhabitants in the neighbourhoods affected was at least four times as much. Whether the routine use of antimicrobial therapy and in particular prophylaxis limited the extent of the cholera epidemic in Douala substantially is difficult to determine. The context did not allow a field trial to be conducted with regards to prophylaxis. This not withstanding, a look at the dynamics of the present epidemic did not show any differences neither in its spatial diffusion and diffusion patterns nor in its seasonal peaking when compared to the relatively well documented 1997/98 cholera epidemic in the same city. Besides, characteristics like the relatively low overall attack rates associated with rather long durations of the cholera outbreaks was similar to what has been observed by Legros et al. (12) and Hutin et al. (13) in other urban settings where systematic prophylaxis was not applied.

In conclusion, the routine use of antimicrobial therapy for cholera cases associated with simultaneous, prolonged, and large scale chemoprophylaxis of close contacts does not seem in our experience to compromise the stability of $V$. cholerae susceptibility profiles to drugs when applied within a comprehensive package of rigorously monitored community interventions. The role of therapy and chemoprophylaxis in limiting the extent of a cholera epidemic is however difficult to ascertain from our experience. Field trials need to be designed to elucidate this aspect.

\section{REFERENCES}

1. Martin C. et un groupe d'experts Recommandations pour Ia pratique de l'antibioprophylaxie en chirurgie. Actualisation. SFAR-ANAES I 999 ; Conférences de consensus. Paris.

2. Sack D.A., Sack R.B., Balakrish Nair G. et al. Cholera. Lancet. 2004; 363: 223-233.

3. Sen Gupta R.G., Sircar B.K., Mondal S., et al. Effects of doxycycline on transmission of Vibrio cholerae infection among family contacts of cholera patients in Calcutta. Bull. WHO. 1978; 56: 323-326. 
4. Garrigue P., Ndayo M., Sicard J.M. et al. Resistance aux antibiotiques des souches de Vibrio cholerae El Tor isolées a Douala (Cameroun). Bull. Soc. Path. Exot. 1986; 79: 305-312.

5. Shears P. Recent developments in cholera. Curr. Opin. Infect. Dis. 2001; 14: 553-558.

6. World Health Organisation. Cholera outbreak. Assessing the outbreak response and improving preparedness. Geneva 2004 (WHO/CPE/ZFK/2004).

7. Guévart E., Soeile J., Mouangue A., et al. Antibiotic susceptibility of Vibrio cholerae: Evolution after prolonged curative and preventive use during the 2004 cholera epidemics in Douala (Cameroon). Med. Mal. Infect. 2006 (in press).

8. Guévart E., Soeile J., Noeske J., Mouangue A. and Fouda A.B. Mass antibiotic prophylaxis against cholera in the New Bell central prison in Douala during the 2004 epidemic. Santé. 2005; 15: 225-227.

9. Ghosh S., Sengupta P.G., Gupta D.N. et al. Chemoprophylaxis studies in cholera: a review of select works. J. Commun. Dis. 1992; 24: 5-57.
10. Société Francaises de Microbiologie. (Coordinateur: Soussy CJ). Recommandations de l'antibiogramme de Ia société franscaise de microbiologie - (2004). http :// sfm.asso.fr/ 10th of July 2006, date last accessed).

11. Champetier de Ribes G., Rakotonjanabelo L.A., Migliani R., et al. Bilan d'un an d'evolution de l'epidemie de cholera a Madagascar de mars 1999 á mars 2000. Cahiers d'etudes et de recherches francophones/ Sante 2000; 10: 277-286.

12. Djadou K., Atakouma D., Agbobli-Apetsiani E., et al. Epidémie de choléra a Dapaong (Togo) de juillet á octobre 1998: cas de l'hopital d'enfants. Med. d'Afrique Noire. 2001; 48: 187-190.

13. Legros D., McCormick M., Mugero C., Skinnider M., Bek'Obita D.D. and Okware S.I. Epidemiology of cholera outbreak in Kampala, Uganda. East Afr. Med. J. 2000; 77: 347-349.

14. Hutin Y., Luby S. and Paquet C. A large cholera outbreak in Kano City, Nigeria: The importance of hand washing with soap and the danger of streetvended water. J. Water Hlth. 2003; 1: 45-52. 